Wien klin Mag 2020 $23: 260-267$ https://doi.org/10.1007/s00740-020-00361-1 Online publiziert: 12 . Oktober 2020 (c) Der/die Autor(en) 2020
Fritz Horak · Barbara Bohle ${ }^{2}$ Gabriele Gadermaier ${ }^{3}$. Wolfram Hötzenecker ${ }^{4}$. Marco Idzko ${ }^{5}$ Verena Niederberger-Leppin ${ }^{6}$. Alexander R. Rosenkranz . Zsolt Szépfalusi ${ }^{8} \cdot$ Gerhard Zlabinger $^{9}$

${ }^{1}$ Allergiezentrum Wien West, Wien, Österreich

${ }^{2}$ Institut für Pathophysiologie und Allergieforschung, Zentrum für Pathophysiologie, Infektiologie und Immunologie, Medizinische Universität Wien, Wien, Österreich

${ }^{3}$ Fachbereich Biowissenschaften, Paris Lodron Universität Salzburg, Salzburg, Österreich

${ }^{4}$ Univ.-Klinik für Dermatologie und Venerologie, Kepler Uniklinikum Linz, Linz, Österreich

${ }^{5}$ Univ.-Klinik für Innere Medizin II, Medizinische Universität Wien, Wien, Österreich

${ }^{6}$ Univ.-Klinik für Hals-, Nasen- und Ohrenkrankheiten, Medizinische Universität Wien, Wien, Österreich ${ }^{7}$ Klinische Abteilung für Nephrologie, Universitätsklinik für Innere Medizin, Medizinische Universität Graz, Graz, Österreich

${ }^{8}$ Univ.-Klinik für Kinder- u. Jugendheilkunde, Medizinische Universität Wien, Wien, Österreich

${ }^{9}$ Institut für Immunologie, Zentrum für Pathophysiologie, Infektiologie und Immunologie, Medizinische Universität Wien, Wien, Österreich

\section{Relevanz homologer Allergene bei der spezifischen Immun- therapie von Pollenallergien}

\section{Statement des Standeskomitees klinische Allergologie der Österreichischen Gesellschaft für Allergologie und Immunologie (ÖGAI)}

\section{Hintergrund}

Die allergische Rhinitis betrifft etwa 20-30 \% der Bevölkerung in Europa [1], wobei viele davon an einer Pollenallergie leiden. Neben der symptomatischen Therapie ist die allergenspezifische Immuntherapie (AIT), auch Hyposensibilisierung genannt, für PatientInnen mit zumindest mittelgradigen Beschwerden eine wichtige Säule des Managements [2]. Die präzise Auswahl der PatientInnen (Indikation, Kontraindikation) ist genauso wichtig wie die Auswahl des relevanten Allergens, der richtigen Applikationsform (subkutan [SCIT], sublingual [SLIT] mit Tropfen oder Allergentabletten) und des adäquaten Produktes mit nachgewiesener Wirksamkeit und Verträglichkeit [3].

Obwohl viele Pollinose-PatientInnen nicht nur gegen eine Allergenquelle (wie Birken- oder Gräserpollen) allergisch sind, ist eine Mischung verschiedener Allergene in einer AIT umstritten. Einer der Hauptgründe dafür ist, dass die meisten Studien nur den Einsatz eines Einzelallergens testen, für welches eine spezifische, therapeutisch wirksame Dosis ermittelt wird. Weiters spricht viel dafür, dass auf der einen Seite die Mischung sehr ähnlicher (sog. homologer) Allergene nicht notwendig ist, auf der anderen Seite die Mischung verschiedener (nicht-homologer) Allergene aufgrund von möglichen Wechselwirkungen zwischen den Allergenen in der Regel nicht empfohlen werden kann [3]. Neben den aktuellen deutschen Leitlinien [3] weist auch die aktuelle europäische Leitlinie darauf hin, dass "Mischungen von Allergenen aus nicht verwandten biologischen Familien für eine allergenspezifische Immuntherapie nicht empfohlen werden können“ [4].
Die Praxis sieht jedoch anders aus. Immer noch werden Mischungen sehr verschiedener Allergene von Pharmafirmen am Markt angeboten, und diese von FachärztInnen zur vermeintlich effektiven AIT bei polysensibilisierten PatientInnen eingesetzt.

Im folgenden Statement werden die verschiedenen Aspekte von homologen und nicht-homologen Allergenen in der Anwendung der AIT bei Pollenallergien erläutert. Im Einzelnen werden homologe Gruppen und deren Referenzallergene beschrieben, die entsprechend den aktuellen Leitlinien zur Diagnostik und Therapie empfohlen werden. In der Folge wird die Relevanz einzelner homologer Pollenallergene für die Immuntherapie im Detail besprochen und praktische Tipps für die Behandlung von PollinosePatientInnen gegeben, die auf mehr als eine Allergenquelle allergisch reagieren. 


\begin{tabular}{|c|c|c|c|c|c|}
\hline Spezies & $\begin{array}{l}\text { Homologe } \\
\text { Gruppe }\end{array}$ & Referenzallergen & $\begin{array}{l}\text { Weitere Vertre- } \\
\text { ter }\end{array}$ & $\begin{array}{l}\text { Homologe } \\
\text { Majorallergene }^{\mathrm{a}}\end{array}$ & Literatur \\
\hline \multirow[t]{9}{*}{ Bäume } & \multirow{6}{*}{$\begin{array}{l}\text { Birken- } \\
\text { Gruppe }\end{array}$} & \multirow[t]{6}{*}{ Birke (Bet v 1) } & Erle & Alng 1 & \multirow[t]{6}{*}{ [10-17] } \\
\hline & & & Hasel & Cor a 1 & \\
\hline & & & Hainbuche & Car b 1 & \\
\hline & & & Eiche & Que a 1 & \\
\hline & & & Edelkastanie & Cas $\mathrm{s} 1$ & \\
\hline & & & Rotbuche & Fag $s 1$ & \\
\hline & \multirow{2}{*}{$\begin{array}{l}\text { Ölbaum- } \\
\text { Gruppe }\end{array}$} & \multirow{2}{*}{$\begin{array}{l}\text { Olivenbaum (Ole e 1) } \\
\text { Esche (Fra e 1) }\end{array}$} & Liguster & Lig $\vee 1$ & \multirow{2}{*}{$\begin{array}{l}{[11,12,} \\
15,18]\end{array}$} \\
\hline & & & Flieder & Syr v 1 & \\
\hline & $\begin{array}{l}\text { Zypressen- } \\
\text { Gruppe }\end{array}$ & $\begin{array}{l}\text { Zypresse (Cup a 1, } \\
\text { Cup s 1) } \\
\text { Zeder (Jun v 1, Jun } \\
\text { a 1) }\end{array}$ & - & - & {$[11,12]$} \\
\hline \multirow[t]{8}{*}{ Gräser } & \multirow{8}{*}{$\begin{array}{l}\text { Süßgräser- } \\
\text { Gruppe }\end{array}$} & \multirow{8}{*}{$\begin{array}{l}\text { Lieschgras (Phl } \\
\text { p 1,2,5) } \\
\text { Knäulgras (Dac } \\
\text { g 1,2,5) } \\
\text { Wiesenrispengras } \\
\text { (Poa p 1,2,5) }\end{array}$} & Ruchgras & Ant o $1,2,5$ & \multirow{8}{*}{$\begin{array}{l}{[11,12,} \\
15 \\
19-23]\end{array}$} \\
\hline & & & Wiesenschwingel & $\begin{array}{l}\text { Fes p } 1,5, \text { e } 1,5 \\
\text { r } 1,5\end{array}$ & \\
\hline & & & $\begin{array}{l}\text { Wolliges Honig- } \\
\text { gras }\end{array}$ & Hol I 1,2,5 & \\
\hline & & & Gerste & Hor $v 1,2,5$ & \\
\hline & & & Lolch & Lol p 1,2,5 & \\
\hline & & & Roggen & $\operatorname{Sec} c 1,2,5$ & \\
\hline & & & Weizen & Tri a $1,2,5$ & \\
\hline & & & Hafer & Ave $s 1,2,5$ & \\
\hline \multirow[t]{3}{*}{ Kräuter } & Beifuß & Beifuß (Art v 1) & - & - & [26] \\
\hline & Ragweed & Ragweed (Amb a 1) & - & - & [24-26] \\
\hline & Sonstige & $\begin{array}{l}\text { Spitzwegerich (Pla } \\
\text { I 1) } \\
\text { Gänsefuß (Che a 1) }\end{array}$ & - & - & [26] \\
\hline
\end{tabular}

\section{Das Prinzip von homologen Allergenen}

\section{Immunologische Aspekte}

Allergieauslösende Pollen enthalten mehrere verschiedene Allergenmoleküle, von denen meist wenige Hauptallergene (Majorallergene) für die allergische Erkrankung verantwortlich sind. Diese Allergenmoleküle sind in der Regel kleine, wasserlösliche Proteine, die typischerweise in ausreichend großer Menge im Pollen vorkommen und anhand ihres molekularen Aufbaus und ihrer Struktur in verschiedene Proteinfamilien zusammengefasst werden [5]. Während der allergischen Sensibilisierung erfolgt die Bildung von IgE-Antikörpern, die spezifisch eine konkrete, kleine Struktur (Epitop) auf der Oberfläche des All-
Antikörper binden grundsätzlich das primär sensibilisierende Allergen (z.B. Bet v 1 aus Birkenpollen) am stärksten. Die beinhaltende Allergenquelle kann somit auch als Referenzallergen bezeichnet werden. Derselbe Antikörper kann an das strukturell ähnliche Epitop eines homologen Allergenmoleküls (wie etwa Cor a 1 im Haselpollen) binden, jedoch typischerweise mit niedrigerer Affinität.

Im Zuge der AIT ist u. a. auch die Produktion von IgE-blockierenden IgG4Antikörpern relevant [9]. Analog zu IgE-Antikörpern erkennen diese ebenfalls Epitope auf Allergenmolekülen und können durch ihre Bindung die Bindung von IgE-Antikörpern an das Allergen verhindern (blockieren). Auch in diesem Fall ist die Stärke der Antikörperbindung an das Referenzallergen, womit AllergikerInnen therapiert wurden, am höchsten. Dennoch kann die Bindung solcher AIT-induzierter Antikörper an homologe Allergene aus anderen Allergenquellen stark genug sein, um die IgE-Bindung an diese zu blockieren und dadurch die IgE-vermittelten Symptome $\mathrm{zu}$ vermindern [10].

\section{Regulatorische Aspekte}

Bis Anfang der 2000er Jahre war es zulässig, Daten von einem Extrakt auf andere Extrakte oder Mischungen zu übertragen, wenn sie zur gleichen taxonomischen Familie gehörten. Ab 2009 wurde das Konzept der homologen Allergene eingeführt [11]. Um zu der gleichen homologen Gruppe zu gehören, müssen die Allergenquellen vergleichbare physikalische, chemische und biologische Eigenschaften erfüllen und von vergleichbaren Geweben stammen (z.B. WeizenmehlAllergene nicht homolog mit WeizenPollen, Weizen-Pollen aber homolog mit Süßgräsern). Darüber hinaus ist der Nachweis einer Kreuzreaktivität zwischen einzelnen Allergenen oder dem Gesamtextrakt in klinischen Antikörperstudien erforderlich [11]. Die European Medicines Agency (EMA) setzte als weitere Bedingung für die Herstellung von Allergenprodukten homologer Gruppen identische Produktionsprozesse und die identische Formulierung des fertigen Produktes voraus [12]. 
Der Vorteil dieses Ansatzes ist es, dass repräsentative Allergene als Vertreter für die jeweilige homologe Gruppe für Diagnostik und Therapie verwendet werden können. Die EMA betont auch, dass unabhängig von der Homologie der Allergene die Anzahl der Extrakte in einer Mischung minimal gehalten werden soll [12].

\section{Relevanz homologer Pollenallergene für die Immuntherapie im Einzelnen}

\section{Baumpollen}

1. Die Birken-Gruppe besteht hauptsächlich aus Vertretern der buchenartigen Gewächse (Fagales) [13]: Birke, Erle, Hasel, Hainbuche und Eiche [11]. Von der EMA wurde diese Liste noch um Edelkastanie [14] und Rotbuche [12] ergänzt. Die einzelnen Vertreter dieser Gruppe zeigen eine hohe Sequenzidentität, Kreuzreaktivität [15] und Übereinstimmung der IgE-Epitope [16] im Vergleich zum Referenzallergen Birke bzw. dessen Majorallergen Bet v 1. Diese immunologischen Beobachtungen werden mittlerweile auch von klinischen Studien gestützt. Eine rezente Studie zeigte in einer Provokationskammer („environmental exposure chamber"), dass eine AIT mit einer Birkenpollentablette BirkenallergikerInnen vor einer Belastung mit Birken sowie (in geringerem Ausmaß) mit Eichen schützt [10]. In einer weiteren, placebokontrollierten Studie an über 600 BirkenpollenallergikerInnen führte eine Birkenpollen-SLIT zu einer signifikanten Verbesserung der allergischen Symptome sowohl in der Birkensaison als auch in der gesamten Baumsaison [17]. Diese Daten implizieren, dass PollenallergikerInnen, die gegen ein Allergen der homologen Birken-Gruppe (•Tab. 1) allergisch sind, mit einer BirkenImmuntherapie ( $\bullet$ Tab. 2 ) behandelt werden können.

2. Zur Ölbaum-Gruppe gehören Ölbaum (Olivenbaum), Esche, Liguster und Flieder. In Österreich ist die Esche der relevanteste Baum dieser

Wien klin Mag 2020 · 23:260-267 https://doi.org/10.1007/s00740-020-00361-1

(c) Der/die Autor(en) 2020

F. Horak · B. Bohle - G. Gadermaier - W. Hötzenecker - M. Idzko • V. Niederberger-Leppin • A. R. Rosenkranz - Z. Szépfalusi · G. Zlabinger

Relevanz homologer Allergene bei der spezifischen Immuntherapie von Pollenallergien. Statement des Standeskomitees klinische Allergologie der Österreichischen Gesellschaft für Allergologie und Immunologie (ÖGAI)

\section{Zusammenfassung}

Eine Allergie gegen Pollen ist die häufigste Indikation für eine allergenspezifische Immuntherapie (AIT). Dabei ist es entscheidend, das richtige Präparat für den jeweiligen Patienten zu wählen. Aus der Forschung wissen wir, dass viele Allergene einzelnen homologen Gruppen zuzuordnen sind (Birken-Gruppe, Gräser etc.). Die Referenzallergene dieser Gruppen können für die Diagnostik und Therapie eingesetzt werden. Eine Mischung homologer Allergene (z. B. Birke + Erle + Hasel) für die AIT ist aus heutiger Sicht daher nicht mehr nötig. Auf der anderen Seite ist eine Mischung nicht-homologer Allergene (z. B. Birke+Gräser + Beifuß) dezidiert nicht zu empfehlen, da dies zu einer Reduktion der Allergenmenge für die einzelnen Allergene führt und es zu möglichen Wechselwirkungen der Extrakte kommen kann, sodass mit schlechteren Erfolgsaussichten der AIT zu rechnen ist. Für die Patienten (Erwachsene, Jugendliche und Kinder) sollen von der verschreibenden Ärztin/dem verschreibenden Arzt die relevanten 1-2 Allergene für die AIT ausgewählt und in separaten Lösungen in jeweils einen Arm appliziert werden. Pharmafirmen sind aufgefordert, auf den Anforderungsformularen die Bestellmöglichkeit nicht-homologer Allergenmischungen zu vermeiden und das Prinzip der homologen Referenzallergene bei der Auswahl der Extrakte zu berücksichtigen.

\section{Schlüsselwörter}

Strukturhomologie $\cdot$ Pollinose $\cdot$ Hyposensibilisierung · Regulatorische Aspekte · Allergenmischung

\section{Relevance of Homologous Allergens in Specific Immunotherapy of Pollen Allergy. Statement of the Standing Commission for Clinical Allergology of the Austrian Society for Allergology and Immunology (ÖGAI)}

\section{Abstract}

Pollen allergy is the most frequent indication for allergen-specific immunotherapy (AIT). Consequently, it is essential to find the right preparation for the patient. As is known from research, several allergens can be assigned to distinct homologous groups (birch group, grasses etc.). The reference allergens from each group can be used for diagnosis and treatment. Therefore, mixing homologous allergens (for example birch + alder + hazel) for AIT is no longer necessary. Furthermore, a mixture of non-homologous allergens (for example birch + grass + mugwort) can definitely no longer be recommended as this will lead to a reduction of allergen content for the single allergen and can cause interaction between the extracts. This can further lead to reduced success rates of AIT. Physicians should select one or two relevant allergens for their patient (adults, adolescents or children) for AIT and inject them in different solutions in one arm each. Pharmaceutical companies have to be requested to avoid the possibility to order non-homologous mixtures on their order forms and to consider the principle of homologous reference allergens in the selection of extracts.

\section{Keywords}

Structural homology · Pollinosis · Hyposensitization - Regulatory aspects - Allergen mixture 
Tab. 2 Entscheidungshilfe, welches diagnostische Allergen für welche spezifische Immuntherapie (AIT) verwendet werden soll

\begin{tabular}{|c|c|c|c|}
\hline \multirow{2}{*}{$\begin{array}{l}\text { Symptome in der jeweiligen } \\
\text { Saison }{ }^{\mathrm{a}} \\
\text { Bäume }\end{array}$} & \multicolumn{2}{|c|}{ Spezifisches IgE } & \multirow[t]{2}{*}{ AIT gegen } \\
\hline & Bet v 1 & Ole e 1 & \\
\hline & + & - & Birke $^{b}$ \\
\hline & - & + & Esche oder Ölbaum \\
\hline & + & + & Birke + Esche oder Ölbaum ${ }^{c}$ \\
\hline Gräser & \multicolumn{2}{|c|}{ Phl p 1,5 } & Lieschgras $^{d}$ \\
\hline \multirow[t]{4}{*}{ Beifuß/Ragweed } & Art v 1 & Amb a 1 & \\
\hline & + & - & Beifuß \\
\hline & - & + & Ragweed \\
\hline & + & + & Beifuß + Ragweed ${ }^{c}$ \\
\hline \multicolumn{4}{|c|}{  } \\
\hline
\end{tabular}

Gruppe und wird neben Olivenbaum als zweites wichtiges Referenzallergen angegeben [11]. Liguster und Flieder sind als weitere Vertreter aufgrund ihrer vorwiegenden Insektenbestäubung in viel geringerem Ausmaß und nur sehr lokal relevant. Eine Studie an österreichischen und spanischen PatientInnen mit einer Olivenbaumbzw. Eschenallergie zeigte eine starke Kreuzreaktivität der Oleaceae zu Ole e 1, welches als Referenzallergen für die Diagnose und Therapie von Oliven- und Eschenpollen-Allergien gesehen werden kann ([18]; - Tab. 1 und 2). Das bedeutet, dass es aus Sicht der Statement-Autoren nicht nur gerechtfertigt ist, eine Eschenpollenallergie mit Hilfe von Ole e 1 zu diagnostizieren, sondern ggf. auch eine AIT gegen Eschenpollenallergie mit Olivenextrakt durchzuführen, sofern beim jeweiligen Präparat Studien zur Wirksamkeit durchgeführt wurden.

3. Die Zypressen-Gruppe umfasst die homologen Allergene der Zeder und Zypresse [11, 12]. Diese spielen in Österreich und Mitteleuropa allerdings eine untergeordnete Rolle.

Andere Baumpollen, die nicht den Gruppen a-c zuzuordnen sind (z.B. Ahorn, Pappel, Robinie, Weide und Ulme) haben ein deutlich geringeres allergenes Potenzial als die o. g. Bäume. Die Platane kann allerdings regional von Bedeutung sein.

\section{Gräserpollen}

Aus allergologischer Sicht spielen unter den Gräserpollen vor allem die Süßgräser (Poaceae) eine wichtige Rolle. Dazu gehören mehrere tausend Spezies, wobei die meisten allergologisch relevanten zur Unterfamilie der Pooideae zählen [19]. Als repräsentative Allergenquellen wurden in Bezug auf regulatorische Maßnahmen Lieschgras, Knäulgras und Wiesenrispengras definiert [11]. Aber viele weitere Gräser- und Getreidepollen gehören zu dieser Gruppe (• Tab. 1). Insbesondere 3 Proteinfamilien (Gräser Gruppe 1, 2, 5) sind für die Gruppenhomologie verantwortlich, wobei auch andere Gruppen allergologisch eine Rolle spielen können. Hejl et al. [20] fanden bei der Untersuchung von PatientInnenSeren vor und nach AIT eine ausgeprägte $\mathrm{T}$ - und $\mathrm{B}$-Zell-Kreuzreaktivität gegenüber Pooideae-Gras-Allergenen unabhängig von der Anzahl der Spezies in den verwendeten Extrakten. Die AutorInnen schlossen daraus, dass eine einzelne Spezies der Süßgräser für eine Allergie-Antwort ausreichend sein dürfte. Auch bei einer Analyse von über 5000 ProbandInnen aus Serumdatenbanken in Nordeuropa und Nordamerika zeigte sich eine ausgeprägte IgE-Kreuzreaktivität zwischen Pooideae-Gräsern (IgE-Inhibition 98-100\%; [21]). Somit könnte ein beliebiges Gras der Pooideae zur Diagnostik und Therapie für die gesamte Gruppe verwendet wer- den. Diese Hypothese wird auch durch klinische Studien mit sublingualen Immuntherapie-Tabletten untermauert, die eine vergleichbare klinische Wirksamkeit sowohl bei der Verwendung einer 5-Gräser-Mischung [22] als auch nur von Lieschgras [23] nachwiesen.

Aus den publizierten Daten kann geschlossen werden, dass Lieschgras (bzw. auch Knäul- oder Wiesenrispengras) als Referenzallergen (•Tab. 1) zumindest für die Diagnose aber auch für die AIT (•Tab. 2) von Allergien gegen Gräseroder Getreidepollen verwendet werden kann.

\section{Kräuterpollen}

$\mathrm{Zu}$ den wichtigsten Vertretern der Kräuterpollen, die allergologisch in Österreich und Mitteleuropa relevant sind, gehören Beifuß (Artemisia vulgaris) und Ragweed (Ambrosia spec.; [24]). Eine untergeordnete Rolle spielen bei uns der Wegerich (Plantago lanceolata), der Gänsefuß (Chenopodium album) und andere Kräuterarten, wie z. B. Glaskraut (Parietaria judaica), welches v.a. im mediterranen Raum vorkommt.

Innerhalb verschiedener RagweedArten besteht eine hohe Kreuzreaktivität. Weiters induzierte eine AIT gegen Ambrosia artemisiifolia (der in Österreich und Europa häufigsten Spezies) auch spezifische IgG-Antikörper gegen andere Ambrosia-Arten [25], die allerdings vermehrt in Amerika und Kanada zu finden sind. Somit kann Ragweed aus allergologischer Sicht zu einer Gruppe zusammengefasst werden. Während in der Publikation von Lorenz et al. [11] noch Beifuß und Ragweed aufgrund der hohen Kreuzreaktivität der Extrakte als gemeinsame Gruppe betrachtet wurden, ergaben neuere Studien ein anderes Bild [26]: Amb a 1, das Hauptallergen von Ragweed ( $>95 \%$ der Ragweed-Allergiker sind dagegen sensibilisiert), zeigt zwar eine hohe Sequenzidentität mit Art v 6, einem homologen Allergen des Beifußes (Pektat-Lyase). Dieses spielt jedoch bei Beifuß-Allergikern eine nur untergeordnete Rolle. Umgekehrt ist bei BeifußAllergikern Art v 1 mit 70-95\% Sensibilisierungsrate das wichtigste Hauptallergen, das mit dem für Ragweed- 


\begin{tabular}{|c|c|c|c|c|c|}
\hline \multicolumn{5}{|c|}{ Klinisch manifeste Allergie gegen } & \multirow[t]{2}{*}{ Mögliche AIT-Kombinationen ${ }^{\text {a }}$ und Therapiebeginn $^{\text {b }}$} \\
\hline Gräser & Birke & Esche & Beifuß & Ragweed & \\
\hline+ & + & - & - & - & Gräser + Birke parallel präsaisonal ${ }^{\complement}$ (Beginn: Sept. bis Dez.) oder ganzjährig \\
\hline+ & - & + & - & - & Gräser + Esche parallel präsaisonal' ${ }^{\complement}$ Beginn: Sept. bis Dez.) oder ganzjährig \\
\hline+ & - & - & + & - & $\begin{array}{l}\text { Gräser + Beifuß parallel ganzjährig, oder gestaffelt präsaisonal` (Gräser: Dez. } \\
\text { bis März, Beifuß: März bis Juni) }\end{array}$ \\
\hline+ & - & - & - & + & $\begin{array}{l}\text { Gräser + Ragweed parallel ganzjährig, oder gestaffelt präsaisonal` (Gräser: } \\
\text { Dez. bis März, Ragweed: April bis Juli) }\end{array}$ \\
\hline- & + & + & - & - & Birke + Esche parallel präsaisonal' (Beginn: Sept. bis Okt.) oder ganzjährig \\
\hline- & + & - & + & - & $\begin{array}{l}\text { Birke + Beifuß parallel ganzjährig, oder gestaffelt präsaisonal }{ }^{\complement} \text { (Birke: Sept. bis } \\
\text { März, Beifuß: März bis Juni) }\end{array}$ \\
\hline- & + & - & - & + & $\begin{array}{l}\text { Birke + Ragweed parallel ganzjährig, oder gestaffelt präsaisonal` (Birke: Sep- } \\
\text { t-März, Ragweed: April-Juli) }\end{array}$ \\
\hline- & - & + & + & - & $\begin{array}{l}\text { Esche + Beifuß parallel ganzjährig, oder gestaffelt präsaisonal }{ }^{\complement} \text { (Esche: Sept. } \\
\text { bis März, Beifuß: März bis Juni) }\end{array}$ \\
\hline- & - & + & - & + & $\begin{array}{l}\text { Esche + Ragweed parallel ganzjährig, oder gestaffelt präsaisonal' (Esche: } \\
\text { Sept. bis März, Ragweed: April bis Juli) }\end{array}$ \\
\hline- & - & - & + & + & Beifuß + Ragweed parallel präsaisonal ${ }^{\complement}$ (Beginn: März/April) oder ganzjährig \\
\hline $\begin{array}{l}{ }^{a} Z \text { u verst } \\
{ }^{\mathrm{b}} \mathrm{Gemäß} \\
\text { at) }\end{array}$ & als getre & pplikati & ier $100 \%$ & $\begin{array}{l}\text { «te (SCIT ode } \\
\text { tsprechende }\end{array}$ & $\begin{array}{l}\text { IT) } \\
\text { Pollensaison (unterschiedlich je nach Region, siehe auch www.pollenwarndienst. }\end{array}$ \\
\hline
\end{tabular}

AllergikerInnen weniger wichtigen Amb a 4 aus derselben Proteinfamilie (Defensin-ähnliche Proteine) eine starke Homologie aufweist. Stemeseder et al. [26] erklären, dass die beobachteten, partiellen Kreuzaktivitäten von Beifuß und Ragweed viele vermeintliche Doppelsensibilisierungen in der Diagnostik erklären können, allerdings nicht für eine homologe Gruppe ausreichend sind. Daher wird heute empfohlen, Amb a 1 und Art v 1 für eine genuine Ragweed bzw. Beifuß-Sensibilisierung (• Tab. 1) als Referenzallergene für die Diagnostik und zum Immuntherapie-Entscheid (- Tab. 2) zu verwenden und nicht als gemeinsame homologe Gruppe anzusehen.

Die Hauptallergene des Spitzwegerichs (Pla 1 1) und des bei uns allergologisch weniger relevanten Gänsefußes (Che a 1) stammen aus der gleichen Olee 1-Familie wie der Ölbaum oder die Esche. Aktuelle Daten zeigten jedoch trotz bestehender moderater Sequenzidentität keine Korrelation auf IgE-Basis zwischen Pla 11 und Ole e 1 [26]. Damit ist Ole e 1 zwar als Referenzallergen für Ölbaum und Esche, nicht aber für Spitzwegerich und Gänsefuß anzusehen (• Tab. 1).

\section{Mischung homologer Allergene}

Bei PollenallergikerInnen, bei welchen die Symptomatik auf ein Referenzallergen einer homologen Gruppe zurückgeführt werden kann (•Tab. 1), ist aus o. g. Gründen die Verwendung des Referenzallergens als Mono-Immuntherapie ausreichend. Prinzipiell wäre eine Mischung verschiedener aus derselben homologen Gruppe stammender Allergene möglich, jedoch nicht nötig. Dort wo diese auch in Studien überprüft sind, spricht nichts gegen eine weitere Verwendung [3] (z. B. Frühblüher-Mix, Gräser-Mix). Eine komplexe Zusammenstellung verschiedener homologer Allergene $\mathrm{zu}$ unterschiedlichen Prozentsätzen (Gräser : Roggen 80\% : 20\% etc.) ist aus Sicht der Autoren jedoch aus o. g. Gründen nicht sinnvoll.

\section{Probleme bei der Mischung nicht-homologer Allergene}

Bei der Mischung von nicht-homologen Allergenen (also Allergenen aus verschiedenen homologen Gruppen) kommt es zu mehreren Problemen:

1. Die kumulative Dosis wird nicht erreicht: Studien zur AIT werden mit fixen Dosierungen und somit definierten Allergenmengen durchgeführt. Eine Mischung mit anderen Extrakten würde zu einer Verdünnung der gemischten Allergene führen und somit möglicherweise zu einer Reduktion der Wirksamkeit der AIT. Eine Anwendung ist nur dann zulässig, wenn auch die jeweilige Allergenmischung in einer Studie geprüft wurde [3].

2. Mögliche Wechselwirkung zwischen den Allergenen: Verschiedene Allergene besitzen proteolytische und andere chemische Eigenschaften (z. B. Milben, Schimmelpilzextrakte) und können daher bei einer Mischung das andere Allergen negativ beeinflussen $[3,12,27]$.

Eine Mischung nicht-homologer Allergene für die Therapie ist aus Sicht der Autoren daher nicht zulässig. Einzige Ausnahme sind Mischungen, deren Wirksamkeit dezidiert in klinischen Studien gezeigt wurde [3]. 


\section{Praktische Tipps für die Be- handlung polysensibilisierter/ polyallergischer Patientinnen}

Die meisten PatientInnen, die im klinischen Alltag (aber auch bei AIT-Studien) betreut werden, sind nicht nur gegen eine einzelne, sondern gegen mehrere Allergenquellen gleichzeitig sensibilisiert [28]. Dabei ist es wichtig, die für die PatientInnen klinisch tatsächlich relevanten Allergene zu identifizieren und mit dem entsprechenden AIT-Extrakt zu therapieren. Eine alleinige Sensibilisierung ohne entsprechende Symptomatik in der jeweiligen Pollensaison ist keine Indikation für eine AIT [3]. Neben der gezielten allergologischen Anamnese, dem Einsatz des Skin-Prick-Tests und der spezifischen IgE-Bestimmung (gegen Gesamtextrakte und Hauptallergene [molekulare Allergiediagnostik]) kommt hier auch der Beschwerdekalender über eine Pollensaison bzw. auch in Einzelfällen eine nasale oder konjunktivale Provokationstestung zum Einsatz [3]. Am Ende sollten (auch aus praktischen Gründen) maximal 2 (Referenz-)Allergene in 2 getrennten Therapielösungen gewählt werden, die in jeweils einen Arm injiziert werden. Auch eine saisonale Staffelung verschiedener Allergene bzw. eine parallele Anwendung von SLIT- und SCITPräparaten ist bei entsprechender Polyallergie möglich (• Tab. 3). Zuletzt besteht auch die Möglichkeit, die AIT zunächst mit einem Allergen zu beginnen und bei entsprechender Restsymptomatik nach 1 oder 2 Jahren zusätzlich ein weiteres relevantes Allergen zu applizieren. Letztere 2 Möglichkeiten bestehen auch, falls wirklich einmal 3 relevante Allergien vorliegen, die einer AIT bedürfen. Alle genannten Empfehlungen sind generell für Erwachsene, Jugendliche und Kinder gleichermaßen zutreffend, sofern die entsprechenden klinischen Studien hierfür vorliegen und eine entsprechende Zulassung besteht.

\section{Conclusio}

Die Pollenallergie ist eine der häufigsten Allergien, mit welcher die AllergiespezialistInnen in der Ordination, im Krankenhaus oder im Allergiezentrum kon- frontiert werden. Die richtige Diagnostik und Therapie ist für die PatientInnen entscheidend.

Die Mischung homologer Allergene in einem AIT-Extrakt ist prinzipiell möglich, aber nicht sinnvoll. Die Mischung nicht-homologer Allergene in einem AIT-Extrakt ist in der Regel aufgrund von Problemen der kumulativen Dosis und möglicher chemischer Interaktionen der Präparate nicht zu empfehlen.

Das Prinzip der homologen Allergene ermöglicht die Definition einzelner homologer Gruppen, die jeweils einem (oder mehreren) Referenzallergen(en) zugeordnet werden können. Dieses Referenzallergen bzw. diese Allergenquelle kann dann sowohl für die Diagnose als auch für die AIT der PatientInnen verwendet werden.

Polysensibilisierte PatientInnen sind von wirklich polyallergischen (mit Hilfe von Anamnese, In-vivo- und In-vitroTests, Beschwerdekalender und ggf. Provokationstests) zu unterscheiden. Für die AIT werden dann die 1-2 relevantesten Allergene ausgewählt und getrennt appliziert.

Pharmafirmen, die AIT-Präparate herstellen, werden aufgefordert, auf den Anforderungsformularen nicht-homologe Mischungen von Pollenallergenen zu vermeiden und das Prinzip der homologen Referenzallergene bei der Auswahl der Extrakte zu berücksichtigen, damit die AIT von niedergelassenen Fachärzten gezielt verordnet werden kann. Mischung von Pollenextrakten mit anderen Allergenquellen (Milben, Schimmelpilze, Tierepithelien etc.) sind allesamt $\mathrm{zu}$ vermeiden.

\section{Korrespondenzadresse}

\section{PD Dr. Fritz Horak}

Allergiezentrum Wien West

Hütteldorferstraße 44-46, 1150 Wien,

Österreich

f.horak@allergiezentrum.at

Univ. Prof. Dr. Zsolt Szépfalusi

Univ.-Klinik für Kinder- u. Jugendheilkunde,

Medizinische Universität Wien

Währinger Gürtel 18-20, 1090 Wien, Österreich

zsolt.szepfalusi@meduniwien.ac.at

Funding. Open access funding provided by Medical University of Vienna.
Einhaltung ethischer Richtlinien

Interessenkonflikt. B. Bohle, G. Gadermaier, M. Idzko, V. Niederberger-Leppin, A.R. Rosenkranz, Z. Szépfalusi und G. Zlabinger geben an, dass kein Interessenkonflikt besteht. F. Horak: Referenten- und Beratungstätigkeit sowie Mitorganisation von Kongressen: ALKAbello, Allergopharma, HAL, Bencard, Stallergenes, LETI. W. Hötzenecker: Referenten und Beratungstätigkeit für ALK-Abello, Bencard, Allergopharma, Novartis.

Für diesen Beitrag wurden von den Autoren keine Studien an Menschen oder Tieren durchgeführt. Für die aufgeführten Studien gelten die jeweils dort angegebenen ethischen Richtlinien.

Open Access. Dieser Artikel wird unter der Creative Commons Namensnennung 4.0 International Lizenz veröffentlicht, welche die Nutzung, Vervielfältigung, Bearbeitung, Verbreitung und Wiedergabe in jeglichem Medium und Format erlaubt, sofern Sie den/die ursprünglichen Autor(en) und die Quelle ordnungsgemäß nennen, einen Link zur Creative Commons Lizenz beifügen und angeben, ob Änderungen vorgenommen wurden.

Die in diesem Artikel enthaltenen Bilder und sonstiges Drittmaterial unterliegen ebenfalls der genannten Creative Commons Lizenz, sofern sich aus der Abbildungslegende nichts anderes ergibt. Sofern das betreffende Material nicht unter der genannten Creative Commons Lizenz steht und die betreffende Handlung nicht nach gesetzlichen Vorschriften erlaubt ist, ist für die oben aufgeführten Weiterverwendungen des Materials die Einwilligung des jeweiligen Rechteinhabers einzuholen.

Weitere Details zur Lizenz entnehmen Sie bitte der Lizenzinformation auf http://creativecommons.org/ licenses/by/4.0/deed.de.

\section{Literatur}

1. Tong MCF, Lin JSC (2016) Chapter 1: Epidemiology of allergic rhinitis throughout the world. In: EAACI Global atlas of allergic rhinits and chronic rhinosinusitis, $\mathrm{S} 61$ (www.eaaci.org)

2. Bousquet J, Pfaar $\mathrm{O}$, Togias $\mathrm{A}$, Schünemann $\mathrm{HJ}$, Ansotegui I, Papadopoulos NG et al (2019) ARIA care pathways for allergen immunotherapy. Allergy 74(11):2087-2210

3. Pfaar O, Bachert C, Bufe A, Buhl R, Ebner C, Eng P et al (2014) Leitlinie zur (allergen-)spezifischen Immuntherapie bei IgE-vermittelten allergischen Erkrankungen. Allergo JInt 23:282

4. Muraro A, Roberts G et al (2017) EAACI Guidelines: Allergen immunotherapy guidelines Part 2: Recommendations. https://medialibrary.eaaci. org/ (@ European Academy of Allergy and Clinical Immunology (EAACI) ISBN 978-3-9524815-1-6). Zugegriffen: 03.09.2020

5. Radauer C, Bublin M, Wagner S, Mari A, Breiteneder $\mathrm{H}$ (2008) Allergens are distributed into few protein families and possess a restricted number of biochemical functions. J Allergy Clin Immunol 121(4):847-852

6. Werfel T, Asero R, Ballmer-Weber BK, Beyer $\mathrm{K}$ Enrique E, Knulst AC, Mari A, Muraro A, Ollert M Poulsen LK, Vieths S, Worm M, HoffmannSommergruber K (2015) Position paper of the EAACI: food allergy due to immunological cross- 


\section{Allergologie}

reactions with common inhalant allergens. Allergy 70(9):1079-1090

7. Aalberse RC, Stadler BM (2006) In silico predictability of allergenicity: from amino acid sequence via 3-D structure to allergenicity. Mol Nutr Food Res 50(7):625-627

8. Matricardi PM, Kleine-Tebbe J, Hoffmann HJ, Valenta R et al (2016) EAACI molecular allergology user's guide. Pediatr Allergy Immunol 27(Suppl 23):1-250

9. Shamji MH, Ljørring C, Francis JN, Calderon MA, Larché $\mathrm{M}$, Kimber I, Frew AJ, Ipsen $\mathrm{H}$, Lund $\mathrm{K}$ Würtzen PA, Durham SR (2012) Functional rather than immunoreactive levels of lgG4 correlate closely with clinical response to grass pollen immunotherapy. Allergy 67:217-226

10. Couroux $P$, Ipsen $H$, Stage BS, Damkjaer JT, Steffensen MA, Salapatek AM, Lund K, Würtzen PA (2019) A birch sublingual allergy immunotherapy tablet reduces rhinoconjunctivitis symptoms when exposed to birch and oak and induces lg G4 to allergens from all trees in the birch homologous group. Allergy 74:361-369

11. Lorenz AR, Lüttkopf D, May S, Scheurer S, Vieths $S$ (2009) The principle of homologous groups in regulatory affairs of allergen products - a proposal. Int Arch Allergy Immunol 148:1-17

12. European Medicines Agency, Committee for medicinal products for human use (2008) Guideline on allergen products: production and quality issues. https://www.ema.europa. eu/documents/scientific-guideline/guidelineallergen-products-production-quality-issues_ en.pdf.Zugegriffen:03.09.2020

13. Kleine-Tebbe J, Zubiet $T$, Werfel T, Krüll $M$, Wagenmann $\mathrm{M}$, Johansen $\mathrm{N}$, Adler Würtzen $\mathrm{P}$, Wolf $H$, Mücke $V$, Wüstenberg $E$, Biedermann $T$ (2020) Is allergy immunotherapy with birch sufficient to treat patients allergic to pollen of tree species of the birch homologous group? Allergy 75(6):1327-1336

14. Hirschwehr R, Jäger $S$, Horak F, Ferreira F, Valenta $R$ Ebner C, Kraft D, Scheiner O (1993) Allergens from birch pollen and pollen of the European chestnut share common epitopes. Clin Exp Allergy 23:755-761

15. Heath MD, Collis J, Batten T, Hutchings JW, Swan N, Skinner MA (2015) Molecular, proteomic and immunological parameters of allergens provide inclusion criteria for new candidates within established grass and tree homologous groups. World Allergy Organ J 8:21

16. Niederberger V, Pauli G, Grönlund H, Fröschl R, Rumpold H, Kraft D, Valenta R, Spitzauer S (1998) Recombinant birch pollen allergens ( $r$ Bet $\mathrm{v} 1$ and rBet v2) contain most of the lgE epitopes present in birch, alder, hornbeam, hazel and oak pollen: a quantitative $\lg \mathrm{E}$ inhibition study with sera from different populations. J Allergy Clin Immunol 102:579-591

17. Biedermann $T$, Kuna $P$, Panzner $P$, Valovirta $E$ Andersson M, de Blay F, Thrane D, Jacobsen $\mathrm{SH}_{4}$ Sonne Stage B, Winther L (2019) The SQ tree SLIT-tablet is highly effective and well tolerated: Results from a randomized, double-blind, placebocontrolled phase III trial. J Allergy Clin Immunol 143(3):1058-1066

18. Palomares O, Swowoda I, Villalba M, Balic N, Spitzauer S, Rodriguez R, Valenta R (2006) The major allergen of olive pollen Ole $\mathrm{e} 1$ is a diagnostic marker for sensitization to oleaceae. Int Arch Allergy Immunol 141:110-118

19. Jaeger $S$ (2008) Exposure to grass pollen in Europe. Clin ExpAllerg Rev 8:2-6
20. Hejl C, Wurtzen PA, Kleine-Tebbe J, Johansen N, Broge L, Ipsen H (2009) Phleum pratense alone is sufficient for allergen-specific immunotherapy against allergy to Pooideae grass pollens. Clin Exp Allergy 39:752-759

21. Johansen N, Weber RW, Ipsen H, Barber D, Broge L, Hejl C (2009) Extensive IgE cross-reactivity towards the pooideae grasses substantiated for al large number of grass-pollen sensitized subjects. Int Arch Allergy Immunol 150:325-334

22. Didier A, Malling HJ, Worm M, HorakF, Sussman GL (2015) Prolonged efficacy of the 300 IR 5-grass pollen tablet up to 2 years after treatment cessation, as measured by a recommended daily combined score. Clin Transl Allergy 5:12-20

23. Valovirta W, Petersen TH, Piotrowska T, Laursen MK, Andersen JS, Sørensen HF, Klink R (2018) Results from the 5-year SQ grass sublingual immunotherapy tablet asthma prevention (GAP) trial in children with grass pollen allergy. J Allergy Clin Immunol 141:529-538

24. Buters JTM, Alberternst B, Nawrath S, Wimme M, Traidl-Hoffmann C, Starfinger U, Behrendt $\mathrm{H}$, Schmidt-Weber C, Bergmann KC (2015) Ambrosia artemisiifolia (ragweed) in Germany-current presence, allergological relevance and containment procedures. Allergo J Int 24:108-120

25. Christensen LH, Ipsen $\mathrm{H}$, Nolte $\mathrm{H}$ et al (2015) Short ragweed is highly cross-reactive with other ragweeds. Ann Allergy Asthma Immunol 115:490-495
26. Stemeseder T, Hemmer W, Hawranek T, Gadermaier G (2014) Markerallergens of weed pollen-basic consideration and diagnostic benefits in the clinical routine. Part 16 of the Series Molecular Allergology. Allergo J Int 23:274-280

27. Nelson HS (2009) Specific immunotherapy with allergen mixes: what is the evidence? Curr Opin Allergy Clin Immunol 9:549-553

28. Calderon MA, Cox L, Casale TB, Moingeon $P$, Demoly $P$ (2012) Multiple-allergen and singleallergen immunotherapy strategies in polysensitized patients: looking at the published evidence. J Allergy Clin Immunol 129:929-934

Hinweis des Verlags. Der Verlag bleibt in Hinblick auf geografische Zuordnungen und Gebietsbezeichnungen in veröffentlichten Karten und Institutsadressen neutral. 
Hier steht eine Anzeige.

\section{曾 Springer}

